\title{
Fusion of Multiple Handwritten Word Recognition Techniques
}

\author{
B. Verma' ${ }^{1}$ and P. Gader ${ }^{2}$ \\ ${ }^{1}$ School of Information Technology $\quad{ }^{2}$ Department of Computer Science \& \\ Griffith University-Gold Coast Engineering \\ PMB 50, Gold Coast Mail Centre University of Missouri-Columbia \\ QLD 9726, Australia \\ Columbia MO 65211,USA \\ E-mail: b.verma@gu.edu.au \\ E-mail: pgader@cecs.missouri.edu
}

\begin{abstract}
Fusion of multiple handwritten word recognition techniques is described. A novel borda count for fusion based on ranks and confidence values is proposed. Three techniques with two different conventional segmentation algorithms in conjunction with backpropagation and radial basis function neural networks have been used in this research. Development has taken place at the University of Missouri and Griffith University. All experiments were performed on real-world handwritten words taken from the CEDAR benchmark database. The word recognition results are very promising and highest $(91 \%)$ among published results for handwritten words.
\end{abstract}

Keywords: Handwritten Word Recognition, Segmentation, Borda Count, Classifier Fusion, Neural Networks, Radial Basis Function, Character Recognition.

\section{INTRODUCTION}

Many successful techniques have been developed to recognize well segmented and isolated handwritten characters and numerals. Excellent recognition results [1-5] have been achieved, however, their success has not carried onto the handwritten word recognition domain [6-13]. This has been ascribed to the difficult nature of unconstrained handwritten words, including the diversity of character patterns, ambiguity and illegibility of characters, and the overlapping nature of many characters in a word [7-8].

Researchers have used different features extraction, segmentation and classification algorithms [6-8, 14-21] to achieve better recognition rates for handwritten words. The results obtained by different techniques vary significantly because many complex procedures such as preprocessing, thinning, slant correction, segmentation and classification are required to recognize unconstrained handwriting. A technique that uses very strict preprocessing and removes noise may recognize some words but it may fail to recognize words that have lost 
information discarded by thinning, slant correction or segmentation. On the other hand, a technique without strict pre-processing or a better segmentation algorithm may recognize those words that were not recognized by the previous technique. Therefore, various techniques in conjunction with conventional and intelligent algorithms make different errors and produce different recognition results. It is very interesting that even if they produce similar results, the mistakes made by them might be different.

Fusion is one of the powerful methods for improving recognition rates produced by various techniques. It takes advantage of different errors produced by different techniques, emphasizes the strengths and avoids weaknesses of individual techniques. Researchers have found [8] that in many real-world applications, it is better to fuse multiple techniques to improve results.

This paper proposes a modified borda count to fuse three techniques developed at two different institutes using different segmentation and neural network algorithms. Experimental results on the CEDAR database from the individual and combined techniques are provided. A comparison of results with conventional borda [8], majority rule [23], averaging [23] and the choquet integral [8] is also included.

The remainder of the paper is broken down into 5 sections. Section 2 describes the proposed technique, Section 3 provides experimental results, a discussion of the results takes place in Section 4 and a conclusion is drawn in Section 5.

\section{PROPOSED TECHNIQUE FOR FUSION}

This section describes the proposed approach to combine three handwritten word recognition techniques (MUMLP, GUMLP, MURBF) using a modified borda count based on ranks and confidence values. An overview of the technique is provided in Figure 1.



\subsection{Conventional Borda Count}

The conventional borda count for a string in a lexicon is defined as the sum of the number of strings that are below the string in the different lexicons produced by the various techniques. For example, if for a string "leonardwood", the top five 
words from the three techniques are as shown in Table 1 below, and the total number of strings is 317 , the borda count can be calculated as follows:

Borda count for "leonardwood" $=316+314+316=946$

Borda count for "ftleonardwood" $=315+316+312=943$

Table 1. Top five words for conventional borda count

\begin{tabular}{|c|c|c|}
\hline $\begin{array}{c}\text { Top five words from } \\
\text { technique 1 }\end{array}$ & $\begin{array}{c}\text { Top five words from } \\
\text { technique 2 }\end{array}$ & Top five words from technique \\
\hline leonardwood & ftleonardwood & 3 \\
ftleonardwood & fortleonardwood & leonardwood \\
fortleonardwood & leonardwood & fortleonardwood \\
flatwood & flatwood & simmons \\
simmons & roubidoux & flatwood \\
roubidoux & simmons & ftleonardwood \\
\hline
\end{tabular}

\subsection{Modified Borda Count}

As can be seen in Section 2.1, the conventional borda count does not take into consideration "the confidence values produced by various techniques" in making the final decision. Also it treats equally all three techniques. In the modified Borda count, we have added three new components as follows:

- Firstly, we assign and use a rank in the calculation of a borda count, instead of calculating the numbers of strings below the string to be recognized. The rank for a particular string can be calculated using the following formula.

Rank=1 -(position of a string in top $\mathrm{N}$ strings $/ \mathrm{N}$ )

The rank is 0 , if the string is not in top $\mathrm{N}$ choices.

$\mathrm{N}=10$ means that only top 10 words are considered from each technique to calculate the rank. Table 2 shows ranks for $\mathrm{N}=5$.

- Secondly and very importantly we use the confidence values produced by different techniques.

Every technique computes a confidence value for each word in the lexicon based on character confidences, compatibility scores, etc. A higher confidence value means that the word is closer to the true word.

- Finally, we use a weight variable for every technique and try to find out the optimum value. It is very similar to the weighted borda count [8] used by some researchers. For certain real-world applications, some techniques may be more accurate than others. We can therefore assign a higher weight to the techniques with higher recognition rates and a low weight to the techniques with lower recognition rates. Instead of assigning a fixed weight value to every technique, there is a better way to find the optimum value. This can be accomplished by varying the weights and selecting the weight values that achieve highest recognition rates. 
Table 2. Ranks and confidence values for top five words for modified borda count

\begin{tabular}{|c|c|c|c|c|c|c|}
\hline \multirow{2}{*}{ Rank } & \multicolumn{2}{|c|}{ Technique 1 } & \multicolumn{2}{c|}{ Technique 2 } & \multicolumn{2}{c|}{ Technique 3 } \\
\cline { 2 - 7 } & Word & $\begin{array}{c}\text { Conf. } \\
\text { value }\end{array}$ & Word & $\begin{array}{c}\text { Conf. } \\
\text { value }\end{array}$ & Word & $\begin{array}{c}\text { Conf. } \\
\text { value }\end{array}$ \\
\hline 1.0 & silver & 47.8 & Silver & 67.4 & silver & 64.2 \\
0.8 & oakhill & 44.5 & Simeon & 43.7 & station & 58.0 \\
0.6 & simeon & 39.8 & Belmont & 37.3 & simeon & 57.3 \\
0.4 & chville & 38.8 & Prince & 34.2 & sanger & 53.2 \\
0.2 & station & 38.2 & Elizcity & 31.4 & fairlea & 51.3 \\
\hline
\end{tabular}

The Modified Borda Count (MBC) can be calculated as follows:

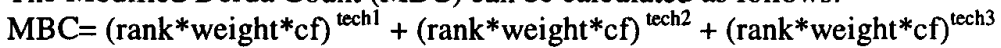

MBC for "silver" $=1 * 0.20 * 47.8+1 * 0.60 * 67.4+1 * 0.20 * 64.2=62.84$

$\mathrm{MBC}$ for "oakhill" $=0.8 * 0.20 * 44.5+0.0+0.0($ rank is 0 if word is not in top 5)=7.12

\subsection{Overview of the MUMLP System}

MUMLP is based on over-segmentation, a multilayer perceptron trained using the backpropagation algorithm and dynamic programming. The segmentation algorithm, confidence assignment, and other details are described well in [6] and we therefore do not discuss them much here. The reader may refer to [6] for more detail about the system. The overview of the MUMLP system is shown in Figure 2.

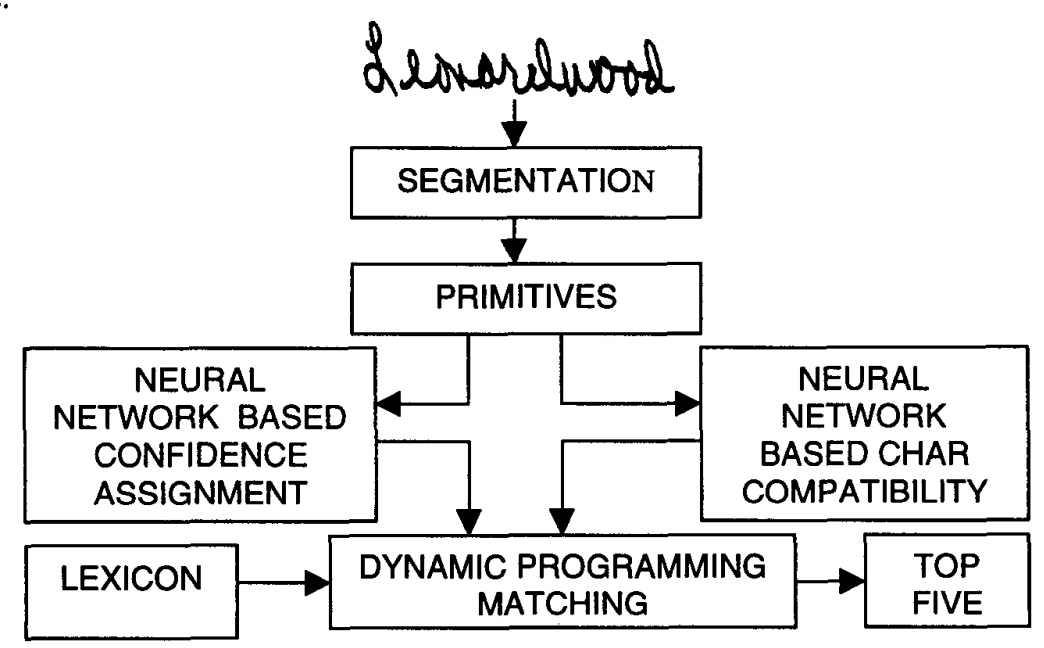

Figure 2. Overview of MUMLP system 


\subsection{Overview of the GUMLP System}

GUMLP is very similar to MUMLP system shown in Figure 2. There are two major differences between these two systems 1) GUMLP is without "NEURAL NETWORK BASED CHAR COMPATIBILITY" component and 2) It uses a recently developed heuristic segmentation algorithm. The reader may refer to [7] for more detail about the system \& the segmentation algorithm.

\subsection{Overview of the MURBF System}

MURBF is based on the radial basis function neural network. The preprocessing, over-segmentation algorithm, dynamic programming, etc, used in MUMLP as shown in Figure 2, were employed in MURBF. Only the neural network component was changed. In MURBF, instead of the backpropagation neural network, a traditional radial basis function neural network was used. After a long investigation based on character and word recognition results using randomly and clustered centers it was found that the 1000 randomly distributed centers was the optimum solution for the CEDAR benchmark database [22]. So in MURBF the randomly distributed 1000 centers were used.

\section{Experimental Results}

The experiments were conducted on handwritten words from the CEDAR CDROM benchmark database [22]. In particular we used words contained in the "BD/cities" directory of the CD-ROM. Some examples of handwritten words used in the experiments are shown below in Figure 3. All of the 317 handwritten city names from the BD directory "test set" were used for testing. The sets of lexicons that have average lengths of 100 words were used. The results of individual techniques are listed in Table 3. The results for combination of techniques are shown in Table 4.

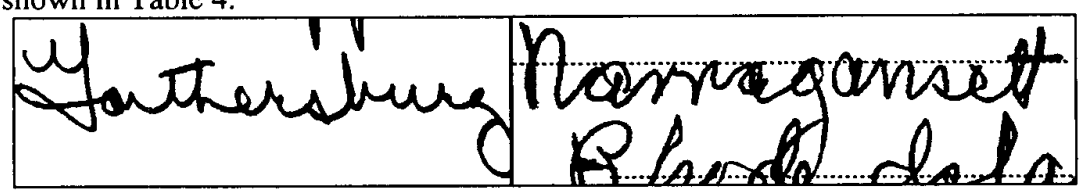

Figure 3. Word samples used for training/testing

Table 3. Word recognition results for individual techniques

\begin{tabular}{||c|c|c|c|c||}
\hline Technique & $\begin{array}{c}\text { Slant } \\
\text { Correction }\end{array}$ & $\begin{array}{c}\text { Preprocessing } \\
\text { /Re-sizing }\end{array}$ & $\begin{array}{c}\text { Character } \\
\text { Compatibility }\end{array}$ & $\begin{array}{c}\text { Recognition } \\
\text { Rate [\%] } \\
\text { Test Set }\end{array}$ \\
\hline GUMLP & Yes [7] & Yes & No & 78 \\
\hline MUMLP & Yes [6] & Yes & Yes [6] & 88 \\
\hline MURBF & Yes [6] & Yes & Yes [6] & 85 \\
\hline
\end{tabular}


Table 4. Word recognition results for combinations of techniques

\begin{tabular}{||c|c|}
\hline Combination Approach & Recognition Rate [\%] Test Set \\
\hline Proposed Borda & 91 \\
\hline Conventional Borda [8] & 88 \\
\hline Majority Rule [23] & 88 \\
\hline Choquet Integral [8] & 84 \\
\hline Averaging [23] & 84 \\
\hline
\end{tabular}

Table 5. Weights for best word recognition results using proposed borda

\begin{tabular}{|c|c|c|c|}
\hline \multicolumn{4}{|c|}{ count } \\
\hline $\begin{array}{c}\text { Recognition } \\
\text { Rate }[\%] \\
\text { Test Set }\end{array}$ & Weights for GUMLP, MUMLP, MURBF \\
\cline { 2 - 4 } & GUMLP & MUMLP & MURBF \\
\hline 91 & 0.20 & 0.60 & 0.20 \\
\hline 91 & 0.18 & 0.54 & 0.18 \\
\hline 91 & 0.02 & 0.06 & 0.02 \\
\hline
\end{tabular}

\section{Discussion}

The results from individual techniques are presented in Table 3. As can be seen, the MUMLP achieved best word recognition results as an individual technique. The reason it achieved the best results was that the MUMLP used compatibility scores and very complicated rules to decide whether a union is valid or invalid during the dynamic programming based matching. Also it used very strict preprocessing which removed all types of noise from words and resized them to a fixed size. GUMLP and MURBF produced lower recognition rates, however during the analysis of results it was found that there were many words (Figure 4) that were not recognized by MLPMU, but were recognized by GUMLP and MURBF.

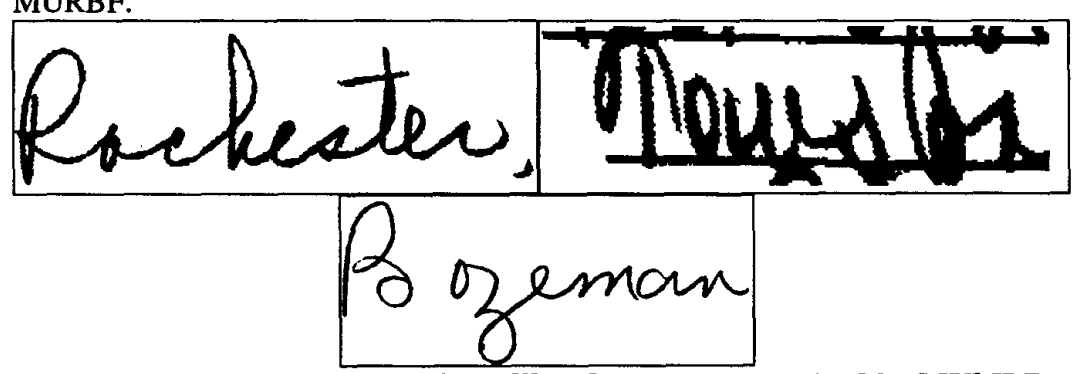

Figure 4. Words recognized by GUMLP and not recognized by MUMLP

The results from combined techniques are presented in Table 4. The proposed borda count achieved the top recognition rate: $91 \%$, which is much better than any 
individual technique and also better than other fusion techniques such as traditional borda count, majority rule [23], averaging [23] and the choquet integral [6]. Modified borda count increased the recognition rate because it takes into consideration confidence values and ranks produced by all three techniques. It is noted that the choquet integral totally failed in our experiments, it decreased the recognition rate instead of increasing it. It is observed, and it can be easily calculated from Tables 3 and 4, that the choquet integral produced results nearly equal to the average of results produced by the three individual techniques. According to our observations, the choquet integral failed because it doesn't give priority to higher confidence values produced by various techniques. The confidence values don't contribute directly to calculating the choquet integral, instead it tries to give a higher weight to the technique with a medium confidence value. It equalizes weights by using the difference between the medium confidence values.

The optimal weights for the three techniques also contributed significantly towards improvement of the recognition rate. To find optimal weight values for the modified borda count, we initialized all three weight variables to zero and then tried to keep one variable stable while incrementing the other two by 0.2 , and repeating the same process for the other two variables. After performing all the experiments, we found that the weighting of MUMLP must be 3 times that of the other two techniques to achieve the best results. The highest weight value for MUMLP is justified because it achieved overall best recognition rate as an individual technique. So it must have greater influence in the final results after combination. The best weight values are shown in Table 4.

The recognition rate is highest among published for handwritten words, however a few words were not recognized by any of the above described three techniques. And it is obvious that those words were not recognized by fusion of three techniques. During the analysis of results it was found that some words (Figure 5) such as "snackouer", "narraagansett", etc., for testing from the CEDAR database were very fuzzy (stamps, lines, two words in one, etc.). We believe that it would be very difficult to recognize such words by any general technique for handwritten words.

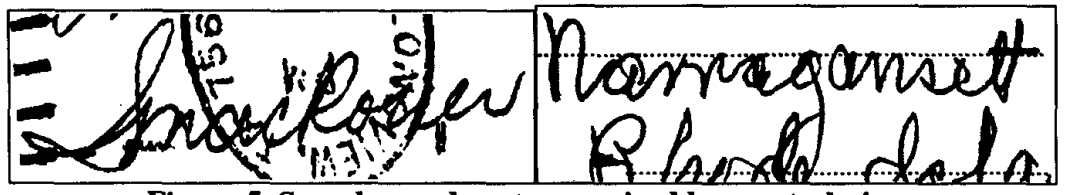

Figure 5. Sample words not recognized by any technique

\section{Conclusion}

Fusion of three different techniques has been presented in this paper, producing excellent results. The main contribution of this paper is a modified borda count for fusion of multiple techniques using the different conventional and intelligent 
algorithms. The conventional borda count, majority rule, averaging, choquet integral and the proposed approach were tested and compared on handwritten words from the CEDAR benchmark database. The borda count proposed in this paper based on word rank and confidence values produced by three different techniques, outperformed other methods.

\section{References}

[1] S-W. Lee, "Multilayer Cluster Neural Network for Totally Unconstrained Handwritten Numeral Recognition", Neural Networks, Vol. 8, 1995, pp. 783792.

[2] H. I. Avi-Itzhak, T. A. Diep, H. Garland, "High Accuracy Optical Character Recognition using Neural Networks with Centroid Dithering", IEEE Trans. Pattern Analysis and Machine Intelligence, Vol. 17, 1995, pp. 218-224.

[3] S-W. Lee, "Off-Line Recognition of Totally Unconstrained Handwritten Numerals Using Multilayer Cluster Neural Network", IEEE Trans. Pattern Analysis and Machine Intelligence, Vol. 18, 1996, pp. 648-652.

[4] S-B. Cho, "Neural-Network Classifiers for Recognizing Totally Unconstrained Handwritten Numerals", IEEE Trans. on Neural Networks, Vol. 8, 1997, pp. 43-53.

[5] M. Gilloux, "Research into the New Generation of Character and Mailing Address Recognition Systems at the French Post Office Research Center", Pattern Recognition Letters, Vol. 14, 1993, pp. 267-276.

[6] P. D. Gader, M. Whalen, M. Ganzberger, D. Hepp, "Handprinted Word Recognition on a NIST Data Set", Machine Vision Applications, Vol. 8, 1995, pp 31-40.

[7] M. Blumenstein, B. K. Verma, Neural-based Solutions for the Segmentation and Recognition of Difficult Handwritten Words from a Benchmark Database, 5th International Conference on Document Analysis and Recognition, Banglore, India, 1999.

[8] P. D. Gader, Magdi A. Mohamed, James M. Keller, "Fusion of Handwritten Word Classifiers", Pattern Recognition Letters, Vol. 17, 1996, pp. 577-584.

[9] C. Y. Suen, R. Legault, C. Nadal, M. Cheriet, L. Lam, "Building a New Generation of Handwriting Recognition Systems", Pattern Recognition Letters, Vol. 14, 1993, pp. 305-315.

[10] S. N. Srihari, "Recognition of Handwritten and Machine-printed Text for Postal Address Interpretation", Pattern Recognition Letters, Vol. 14, 1993, pp. 291-302.

[11] R. M. Bozinovic, S. N. Srihari, "Off-Line Cursive Script Word Recognition", IEEE Trans. Pattern Analysis and Machine Intelligence, Vol. 11, 1989, pp. 68-83.

[12] B. A. Yanikoglu, P. A. Sandon, "Off-line cursive handwriting recognition using style parameters", Tech. Report PCS-TR93-192, Dartmouth College, NH., 1993. 
[13] J-H. Chiang, "A Hybrid Neural Model in Handwritten Word Recognition", Neural Networks, Vol. 11, 1998, pp. 337-346.

[14] R. G. Casey, E. Lecolinet, "A Survey of Methods and Strategies in Character Segmentation", IEEE Trans. Pattern Analysis and Machine Intelligence, Vol. 18, 1996, pp. 690-706.

[15] N.W. Strathy, C.Y. Suen, A. Krzyzak, "Segmentation of Handwritten Digits using Contour Features", ICDAR '93, 1993, pp. 577-580

[16] G. L. Martin, M. Rashid, J. A. Pittman, "Integrated Segmentation and Recognition through Exhaustive Scans or Learned Saccadic Jumps", Int'l J. Pattern Recognition and Artificial Intelligence, Vol. 7, 1993, pp. 831-847.

[17] B. Eastwood, A. Jennings, A. Harvey, "A Feature Based Neural Network Segmenter for Handwritten Words", Int'l Conf. Computational Intelligence and Multimedia Applications, Gold Coast, Australia, 1997, pp. 286-290.

[18] Y. Lu, M. Shridhar, "Character Segmentation in Handwritten Words - An Overview", Pattern Recognition, Vol. 29, 1996, pp. 77-96.

[19] N. Otsu, "A threshold selection method from gray level histograms", IEEE Trans. Systems, Man and Cybernetics, Vol SMC-9, 1979, pp. 62-66.

[20] K. Han, I. K. Sethi, "Off-line Cursive Handwriting Segmentation", ICDAR '95, Montreal, Canada, 1995, pp. 894-897.

[21] B. Yanikoglu, P. A. Sandon, "Segmentation of Off-line Cursive Handwriting using Linear Programming", Pattern Recognition, Vol. 31, 1998, pp. 18251833.

[22] J. J. Hull, "A Database for Handwritten Text Recognition", IEEE Transactions of Pattern Analysis and Machine Intelligence, Vol. 16, 1994, pp. 550-554.

[23] A. Verikas, A. Lipnickas, K. Malmqvist, M. Bacauskiene, A. Gelzinis, "Soft combination of neural classifiers: A comprative study", Pattern Recognition Letters, Vol. 20, 1999, pp. 429-444. 